\title{
Development of novel simple sequence repeat markers from a genomic sequence survey database and their application for diversity assessment in Jatropha curcas germplasm from Guatemala
}

\author{
R.S. Raposo ${ }^{1 *}$, I.G.B. Souza ${ }^{2 *}$, M.E.C. Veloso ${ }^{2}$, A.K. Kobayashi ${ }^{3}$, \\ B.G. Laviola ${ }^{3}$ and F.M. Diniz ${ }^{2}$ \\ ${ }^{1}$ Núcleo de Biologia Experimental, Universidade de Fortaleza, \\ Fortaleza, CE, Brasil \\ ${ }^{2}$ Embrapa Meio-Norte, Teresina, PI, Brasil \\ ${ }^{3}$ Embrapa Agroenergia, PqEB, Brasília, DF, Brasil \\ *These authors contributed equally to this study. \\ Corresponding author: F.M. Diniz \\ E-mail: fabio.diniz@embrapa.br
}

Genet. Mol. Res. 13 (3): 6099-6106 (2014)

Received July 29, 2013

Accepted December 9, 2013

Published August 7, 2014

DOI http://dx.doi.org/10.4238/2014.August.7.25

\begin{abstract}
The last few years have seen a significant increase in the number of large-scale sequencing projects generating whole genome databases. These sequence databases can be surveyed (genome sequence survey) for tandem repeats as an alternative means to develop microsatellites for monitoring and selecting natural populations and cultivars of Jatropha curcas. A total of 100 tandem repeats were revealed from mining 368 genomic surveyed sequences available in the Kazusa DNA Research Institute database. Twenty microsatellite sequences were successfully amplified, resulting in repeatable and scorable polymerase chain reaction products. Genotyping of J. curcas
\end{abstract}


accessions from the Guatemalan population revealed 18 polymorphic loci. The average number of alleles per locus was 6.9, and allelic sizes ranged from 94 to $299 \mathrm{bp}$. Expected and observed heterozygosities ranged from 0.118 to 0.906 and from 0.082 to 0.794 , respectively. Polymorphic information content values ranged from 0.114 (JcSSR-34) to 0.886 (JcSSR-33) with an average of 0.627. Analysis with MicroChecker indicated few null alleles for locus JcSSR-37 in Guatemalan populations, which may be a possible cause of its deviation from Hardy-Weinberg equilibrium, even after Bonferroni's correction. No loci showed significant linkage disequilibrium. These microsatellite loci are expected to be valuable molecular markers in J. curcas because they show high levels of polymorphism and heterozygosity.

Key words: Microsatellites; Energy crop; Physic nut; Biofuel; Euphorbiaceae; Genetic diversity

\section{INTRODUCTION}

Jatropha curcas is a plant with possible origin in Central America and is currently distributed in almost all regions of the world (Fairless, 2007; Dias et al., 2012). Its commercial interest has been sparked by its strong potential in the production of alternative fuels, which is mainly due to its high oil yield (Berchmans and Hirata, 2008). The species exists in climates that are unfavorable for most food crops, and it occurs spontaneously in very poor fertility soils (Arruda et al., 2004). Because of this, J. curcas can be considered as one of the most promising alternative oil sources to replace petroleum diesel. The plant is very resistant to disease, insect attack, mollusks, and fungi because it segregates caustic latex that drips from damaged or torn leaves (Emeasor et al., 2005; Thomas et al., 2008).

Along with the growing interest in the exploitation and commercial cultivation of $J$. curcas, the development of molecular technologies for monitoring and selecting natural populations and cultivars is of paramount importance. The sustainability of the activity must go through the implementation of molecular techniques based on DNA analysis.

In this context, molecular markers such as microsatellites have been widely used (DeWoody et al., 1995; Ellegren, 2004; Guo et al., 2013; Qi et al., 2013). Microsatellites are repeated sequences of nucleotides that are distributed throughout the genome and occur mainly in non-coding regions (Ellegren, 2004). These sequences are used as markers because of their highly polymorphic pattern. Microsatellites can be used not only in the conservation of genetic resources of commercial interest but also in the selection of plants with desirable features for cultivation and identification of quantitative trait loci related to diseases or reproduction (Nambisan, 2007).

The last few years have seen a significant increase in the number of large-scale sequencing projects generating whole genome databases. These sequence databases can be surveyed (genome sequence survey) for tandem repeats as an alternative means to develop microsatellites, which may represent a useful and low-cost procedure for discovering novel microsatellite markers in a genome. Now that the whole genome of J. curcas has been sequenced (Sato et al., 2011), it is valuable to use the database for mining microsatellite loci in the species. 
Thus, this study aimed at mining part of the $J$. curcas genome database to increase the availability of microsatellites, which are hereafter intended for the genetic analysis of $J$. curcas worldwide, and provide a genetic snapshot of the Guatemalan population diversity.

\section{MATERIAL AND METHODS}

\section{Identification of repeated regions and primer design}

A total of 368 contig consensus sequences were downloaded from the Kazusa DNA Research Institute database (www.kazusa.or.jp/jatropha/). These surveyed sequences were screened for tandem repeats using the Tandem Repeat Occurrence Locator software (Castelo et al., 2002). Primer pairs for microsatellite loci were designed based on the unique flanking regions of each repeat (dinucleotide, trinucleotide, tetranucleotide, and pentanucleotide) by using PRIMER 3 with the default settings (Rozen and Skaletsky, 2000) but with a fragment length limit of $300 \mathrm{bp}$ (Table 1). The presence of multiple tandem repeats at distant sites in the same contig sequence allowed the design of different primer pairs targeted to each microsatellite. Only one pair of primers was designed for contiguous microsatellites, even if they were separated by a few bases.

\section{Plant material and DNA isolation}

Fresh young plant leaves of $J$. curcas were collected from the Biocombustibles de Guatemala S.A. germplasm bank and stored in a plastic bag with silica gel to dehydrate. Approximately $20 \mathrm{mg}$ plant tissue was ground in $2.0-\mathrm{mL}$ microtubes containing ceramic grinding beads (CK28, BioAmerica, USA) with two 16-s pulses at $5200 \mathrm{rpm}$ with 10 -s intervals on a Precellys ${ }^{\circledR} 24$ Tissue Homogenizer (Bertin Technologies, France). These homogenized materials were used for DNA isolation with DNeasy Plant Mini Kit (Qiagen, Germany), according to manufacturer instructions, and checked for quality and quantity on a $1 \%$ agarose gel with ethidium bromide. The DNA concentration was measured using a NanoDrop 2000 Spectrophotometer (Thermo Scientific, USA). DNA was maintained at $-20^{\circ} \mathrm{C}$ until further analyses.

\section{Polymerase chain reaction (PCR) amplification and microsatellite marker polymorphism}

Forty-nine accessions of $J$. curcas from Guatemala were used for the characterization of microsatellite markers. PCR amplifications and optimization of primers were performed in a total reaction volume of $10 \mu \mathrm{L}$ consisting of $1.0 \mu \mathrm{L}$ template DNA (20-100 ng), $1.5-2.5 \mathrm{mM} \mathrm{MgCl}_{2}$, $50 \mu \mathrm{M}$ of each dNTP, $0.5 \mathrm{U}$ Taq DNA polymerase (RBC), 0.3-0.5 $\mu \mathrm{M}$ forward and reverse primers, and $1 \mathrm{X}$ PCR buffer $(10 \mathrm{mM}$ Tris-HCl, $\mathrm{pH} 8.3,50 \mathrm{mM} \mathrm{KCl})$ in a Veriti ${ }^{\circledR}$ 96-well thermalcycler (Applied Biosystems, USA). The cycling conditions included an initial denaturation cycle at $95^{\circ} \mathrm{C}$ for $2 \mathrm{~min} ; 30$ cycles of $95^{\circ} \mathrm{C}$ for $45 \mathrm{~s}, 30 \mathrm{~s}$ for annealing (temperature depended on each primer pair; Table 2), and $45 \mathrm{~s}$ for extension at $72^{\circ} \mathrm{C}$; and a final extension at $72^{\circ} \mathrm{C}$ for $10 \mathrm{~min}$.

Microsatellite markers were screened on denaturing $6 \%$ polyacrylamide gels that were stained with silver nitrate for genotype scoring. Allele sizes were initially determined against a 10-bp DNA ladder (Invitrogen, USA) and by comparison with the expected size of the cloned fragment, and then they were scored manually. If necessary, two or more runs were performed to verify the allele typing by re-ordering the samples. 


\section{Statistical analysis}

The numbers of alleles at each microsatellite locus, the proportion of individual samples that are heterozygous (observed heterozygosity), the estimate of heterozygosity (expected heterozygosity), and tests for departure from Hardy-Weinberg equilibrium (HWE) were performed using the probability test in GENEPOP version 3.3 (Raymond and Rousset, 1995). Allele frequencies obtained from the microsatellite genotypes were also used to calculate the polymorphic information content (PIC) of each locus using Cervus 3.0 (Kalinowski et al., 2007) in order to measure the degree of polymorphism obtained by a microsatellite. The occurrence of linkage disequilibrium between loci and the allelic richness for each locus were calculated using FSTAT 2.9.3 (Goudet et al., 1995). Significance levels were adjusted using sequential Bonferroni's corrections. Micro-Checker v2.2.3 was used to determine the most probable cause of any deviation from HWE (Van Oosterhout et al., 2004).

\section{RESULTS AND DISCUSSION}

A total of $100(27.17 \%)$ tandem repeats were revealed from mining 368 genomic surveyed sequences (contigs) available in the Kazusa DNA Research Institute database for $J$. curcas. From these microsatellite-containing sequences, $71 \%$ were dinucleotide repeats, $28 \%$ were trinucleotides, and $1 \%$ were tetranucleotides. Because primer designing was not considered for flanking regions of microsatellites with a small number of repeats, as low levels of variability or fixation is expected (Amos, 1999), our efforts focused on provisional markers with more than 8 tandem repeats. Additionally, other repeat regions were also found that were too short or had GC content that was too low to have primers designed on their flanks. Twenty microsatellite sequences were successfully amplified, resulting in repeatable and scorable PCR products.

Of 20 loci, 18 (90\%) contained dinucleotide microsatellites, of which, $16(88.9 \%)$ were pure, with only two other sequences that were characterized as compound dinucleotides. Two loci were trinucleotide repeats (Table 1).

Genotyping of $J$. curcas accessions from the Guatemalan population revealed 18 polymorphic loci; however, primers for locus JcSSR-38b were merely designed as an amplification alternative. The other two loci were monomorphic (Table 2).

The average number of alleles per locus was 6.9, ranging from 3 (JcSSR-38a/b and JcSSR-42) to 16 (JcSSR-33). The allelic sizes ranged from 94 to $299 \mathrm{bp}$. Expected and observed heterozygosities ranged from 0.118 to 0.906 and from 0.082 to 0.794 , respectively. Distributions of allele frequencies in the $J$. curcas population are shown in Figure 1. Mean expected and observed heterozygosities were 0.675 and 0.593 . PIC values ranged from 0.114 (JcSSR-34) to 0.886 (JcSSR-33) with an average of 0.627 . This genetic parameter is an important estimate of the extent of polymorphism of the marker (DeWoody et al., 1995). PIC values higher than 0.5 are considered to be highly informative. PIC values between 0.25 and 0.50 indicate a reasonably informative locus (Botstein et al., 1980). Therefore, all the analyzed microsatellites were informative in the Guatemalan population of J. curcas except marker JcSSR-34 (PIC: 0.114). Analysis with Micro-Checker indicated few null alleles for locus JcSSR-37 in Guatemalan populations, which may be a possible cause of its deviation from HWE, even after Bonferroni's correction for multiple comparisons at the 5\% significance level (critical value for $\mathrm{P}>0.0029$ ). No loci showed significant linkage disequilibrium after Bonferroni's correction.

These additional microsatellite loci described here are expected to be valuable molecular markers because they show high levels of polymorphism and heterozygosity. 
Table 1. Microsatellite marker information based on the candidate sequence.

\begin{tabular}{|c|c|c|c|c|}
\hline Marker name & Contig code & Contig sequence & Motif & Fragment length (bp) \\
\hline JcSSR-24 & JCCA0100471 & $\begin{array}{l}\text { cgctgtaggaatggcatgttatcaaaataataataataataataataataatg } \\
\text { aaatgaatgaaaaatgataaaaaaagaaagagagaatgattgcttc }\end{array}$ & $(\mathrm{AAT})_{9}$ & 100 \\
\hline JcSSR-25 & JCCA0100841 & $\begin{array}{l}\text { cttggtgttgggettacattgtcatctaaaccaagcatcatgtgtgcttgcttt } \\
\text { atgaattatgatatgtatatatatatatatatatatatatatgtaaattactgggta } \\
\text { tagaattctctgtagtttgtttactatctaatttgttttgctgtttgtataacttttca } \\
\text { gattcagattctggcagctcatctggcagtgaatcagatgcagctaaagctt } \\
\text { cagttcctagtgctgtgacc }\end{array}$ & $(\mathrm{TA})_{13}$ & 243 \\
\hline JcSSR-26 & JCCA0101161 & $\begin{array}{l}\text { aatctactatcccgcatgaaatttttgcaaatttatatgatttaaaatattcaaa } \\
\text { acaaaatattgtcccaatatatattttgtctatatatatatatatatatatatata } \\
\text { tatattgatcacatttggaaattgccacagaatggagaaagagtagga }\end{array}$ & $(\mathrm{AT})_{15}$ & 160 \\
\hline JcSSR-27 & JCCA0101071 & $\begin{array}{l}\text { ctgcatctgggaaacaaattttatgtcatatatatatatatatatatatatatatatatacac } \\
\text { gcacacatatatcttttaaggaccgtcattatttttgataggcatgggtgttat }\end{array}$ & $(\mathrm{AT})_{16}$ & 118 \\
\hline JcSSR-28 & JCCA0101741 & $\begin{array}{l}\text { tatgcaagtacggttgtgtaaccttccattctaatgaaaaactctctctctctc } \\
\text { tctctctctctctctcaacattaaagtttgttctgaaaagccagaaaattattac } \\
\text { tcagaacatcacaacttagtacagaattattagattcctcaagatttaatagct } \\
\text { tgttaaacttttagacattaccataaccaagaattagctg }\end{array}$ & $(\mathrm{CT})_{14}$ & 203 \\
\hline JcSSR-29 & JCCA0101021 & $\begin{array}{l}\text { aaaatagaaaataaaactcgctcaaatatatatatatatatatatatatatatatatatata } \\
\text { taatataaaaatttatgatataatatgttataattgcaaaccatacaatacattg }\end{array}$ & $(\mathrm{TA})_{19}$ & 117 \\
\hline JcSSR-30 & JCCA0101201 & $\begin{array}{l}\text { gtgcgcccaaatgaaaaataaggaaaatattatttccttcaatattttcctcttatttt } \\
\text { cttcaatattatatatatatatatatatatatatatatatatatatatatatatatatta } \\
\text { tttttatattatagtattataaaatgcttatttatattgctaaaataaaattaaatgt } \\
\text { taaataaaaagatacattgtttgttggcttaatgtgtaaaaatactcctaattac } \\
\text { gacttattgcaaaatcatcctaagacgaatattttgcccetggcaaccccat }\end{array}$ & $(\mathrm{AT})_{24}$ & 286 \\
\hline JcSSR-31 & JCCA0102251 & $\begin{array}{l}\text { atttgattgattgccegacggatgggctgtatgtattaatatatatatatatatatata } \\
\text { atattattgtgatatttaaacaggtagatagtcctattttaggggaggtgctgc }\end{array}$ & $(\mathrm{TA})_{10}$ & 114 \\
\hline JcSSR-32 & JCCA0102911 & $\begin{array}{l}\text { tcttttgactacccaatgaccaacccatctgeccectcgcattgttaggagc } \\
\text { gccccaaatttcaccettctctctctctctctctctcttcattttagaatcaccg } \\
\text { gtttactgaaccaccggtttggttcattaaaattaccaaaattagcttcattac } \\
\text { ttaatttcaaccaactctagcacga }\end{array}$ & $(\mathrm{CT})_{10}$ & 188 \\
\hline JcSSR-33 & JCCA0103351 & $\begin{array}{l}\text { gagaacgtggagagttaaaaattttaaattttatcttttcgacttaatattatttt } \\
\text { aacctattgacttttctagtaaattatatgtaaaagattcaaaatgttatatatat } \\
\text { atatatatatatatatatatatatatatatatatatatatatatatatttaaataatta } \\
\text { taaagaaacaaaataaatatttttttagttttgtaaaattgaaattccaaatcct } \\
\text { agttgattgtttttcettatgaagcagacaatgatccatcca }\end{array}$ & $(\mathrm{TA})_{28}$ & 269 \\
\hline JcSSR-34 & JCCA0103431 & $\begin{array}{l}\text { ctaaaagttttggacatttttactaaatattttattattattattattattattattaaaaaga } \\
\text { gtcacaattggtttagtttgaataaaaaatatctgattagacaaaagattgata } \\
\text { gaaattaatttcaaaatttatattgagaatataattacaggagtgcttccattaa }\end{array}$ & $(\mathrm{TTA})_{9}$ & 174 \\
\hline JcSSR-35 & JCCA0103981 & $\begin{array}{l}\text { gagtttggttggatggatatttaagtttgagtgtgtatatatatatatatatatat } \\
\text { atatatatatatatatatatatatatatatatatatatatatatgactttttaaatca } \\
\text { aatagttaatttagtttctttttttaccttatttcactaatactttgttgccattagc } \\
\text { tagaaataatgtgaataaaatttctgctgcggttcta }\end{array}$ & $(\mathrm{TA})_{32}$ & 210 \\
\hline JcSSR-36 & JCCA0104571 & $\begin{array}{l}\text { gcactcaaaagtgtttcccaaaaaataccccaaattatcttcactatttttctc } \\
\text { taccctagttttagaaccctttctaaaacacactctctctctctctctctctctct } \\
\text { ctctctctctctctctctctatatatatatatatatagatatatgctcatgccetgc } \\
\text { attcttctttggtgtgat }\end{array}$ & $(\mathrm{CT})_{22}$ & 185 \\
\hline JcSSR-37 & JCCA0010541 & $\begin{array}{l}\text { tgcaggcacaccaaacaattaaatatcgtgatgcaagtgtataattagacat } \\
\text { tcatttcaattacaattaaataaaggagtagtaattaacaatacaatataata } \\
\text { attgatccttggaaaaaaataaataataaaataataaaataataataaagtaa } \\
\text { atcaagaacttcatttcaatatatatatatatatatatatatatatatatatata } \\
\text { tatatatatagttaaagaattctatctaaataaacctattaacttaattattaatta } \\
\text { tgactaacctagattccatcgaaa }\end{array}$ & $(\mathrm{AT})_{23}$ & 295 \\
\hline JcSSR-38 & JCCA0105851 & $\begin{array}{l}\text { tgaagctgtctttgattgaggaaaaagcttcttcactgctacagtcaaatctg } \\
\text { gctgcaattgagcctataatggagggaataaaatattaaagaaaatgatc } \\
\text { agcaactccttaatgccaatatatatatatatatatatatatatatatgtgtgtgt } \\
\text { gtgtgtgtgtgtgtgtaaagacagagctagaccttgtagacgatcccaaga }\end{array}$ & $(\mathrm{AT})_{15}(\mathrm{GT})_{12}$ & 211 \\
\hline JcSSR-38b & & $\begin{array}{l}\text { atcagcaactcettaatgccaatatatatatatatatatatatatatatatgtgtg } \\
\text { tgtgtgtgtgtgtgtgtgtaaagacagagctagaccttgtagacgatccca } \\
\text { agacggccatctccaatcttcgtttttgggctgaacttttgagtt }\end{array}$ & $(\mathrm{AT})_{15}(\mathrm{GT})_{12}$ & 153 \\
\hline
\end{tabular}

Continued on next page 


\begin{tabular}{|c|c|c|c|c|}
\hline Marker name & Contig code & Contig sequence & Motif & Fragment length (bp) \\
\hline JcSSR-39 & JCCA0001061 & $\begin{array}{l}\text { ttttaacatattgggttgaattttactctatatatatatatatatatataaagttattact } \\
\text { acagatgaaaatgataaacctagtccttctataccttctgttatttatgcaaaa }\end{array}$ & $(\mathrm{TA})_{11}$ & 115 \\
\hline JcSSR-40 & JCCA0107961 & $\begin{array}{l}\text { agtggtggttgtgetcatagtgctattcctcataattgttcctaagaatgttg } \\
\text { atatatatatatatatatatatataaatttgcaaagaaaattaaatcattttaatt } \\
\text { aatttagctttaattaaatttcaatgagaatattctaattgtatataaaatgatttt } \\
\text { cacacatagatatggatagcgtgacccac }\end{array}$ & $(\mathrm{AT})_{12}$ & 196 \\
\hline JcSSR-41 & JCCA0010831 & $\begin{array}{l}\text { gtacgagcgtggactaacttcacctgatgtgtgtcggtgctgctaaaatcat } \\
\text { gcatgacgtggggccattgtatgetccaagccgtcacatttaccgectatat } \\
\text { atatatatatatatatatatatatatatatatatatatcttcegtttcaaaatatttgt } \\
\text { aatgtcaatttttttaaaaacacattaattgtattctcataatatataaaatatcta } \\
\text { tatttttctaaattgtcetcctttaa }\end{array}$ & $(\mathrm{TA})_{21}$ & 169 \\
\hline JcSSR-42 & JCCA0108601 & $\begin{array}{l}\text { tgcatcaacacatccattcttccaccaatatatatatatatatatataaagaa } \\
\text { aactctgagataaaattctgcaataacttctcaatcttgctgaattagaaatca } \\
\text { agttgaaattccaaatgctacttcccactc }\end{array}$ & $(\mathrm{AT})_{10}$ & 138 \\
\hline
\end{tabular}

Table 2. Characteristics of 20 genome sequence survey-derived microsatellites for Jatropha curcas.

\begin{tabular}{|c|c|c|c|c|c|c|c|c|c|}
\hline Marker name & & $\mathrm{Ta}\left({ }^{\circ} \mathrm{C}\right)$ & Size range (bp) & $N_{\mathrm{A}}$ & $H_{\mathrm{E}}$ & $H_{\mathrm{o}}$ & HWE & $F_{\text {IS }}$ & PIC \\
\hline JcSSR-24 & $\begin{array}{l}\text { F: CGCTGTAGGAATGGCATGTT } \\
\text { R: CAAGCAATCATTCTCTCTTTCTTTT }\end{array}$ & 60 & 100-109 & 4 & 0.691 & 0.714 & 0.0040 & -0.033 & 0.625 \\
\hline JcSSR-25 & $\begin{array}{l}\text { F: CTTGGTGTTGGGCTTACATT } \\
\text { R: GGTCACAGCACTAGGAACTGA }\end{array}$ & 62 & 231-249 & 4 & 0.698 & 0.592 & 0.2563 & 0.152 & 0.630 \\
\hline JcSSR-26 & $\begin{array}{l}\text { F: AATCTACTATCCCGCATGAA } \\
\text { R: TCCTACTCTTTCTCCATTCTGT }\end{array}$ & 58 & $136-160$ & 6 & 0.791 & 0.689 & 0.8493 & 0.129 & 0.751 \\
\hline JcSSR-27 & $\begin{array}{l}\text { F: CTGCATCTGGGAAACAAAT } \\
\text { R: ATAACACCCATGCCTATCAA }\end{array}$ & 57 & $106-128$ & 4 & 0.430 & 0.364 & 0.0878 & 0.153 & 0.390 \\
\hline JcSSR-28 & $\begin{array}{l}\text { F: TATGCAAGTACGGTTGTGTA } \\
\text { R: CAGCTAATTCTTGGTTATGG }\end{array}$ & 57 & 197-207 & 5 & 0.611 & 0.531 & 0.0053 & 0.132 & 0.556 \\
\hline JcSSR-29 & $\begin{array}{l}\text { F: AAAATAGAAAATAAAACTCGCTCAAA } \\
\text { R: CAATGTATTGTATGGTTTGCAATTA }\end{array}$ & 56 & $94-128$ & 13 & 0.876 & 0.794 & 0.0087 & 0.093 & 0.848 \\
\hline JcSSR-30 & $\begin{array}{l}\text { F: GTGCGCCCAAATGAAAA } \\
\text { R: ATGGGGTTGCCAGGG }\end{array}$ & 57 & $254-288$ & 7 & 0.805 & 0.750 & 0.4681 & 0.069 & 0.768 \\
\hline JcSSR-31 & $\begin{array}{l}\text { F: ATTTGATTGATTGCCCGAC } \\
\text { R: GCAGCACCTCCCCTAAAA }\end{array}$ & 59 & 114 & 1 & - & - & - & - & - \\
\hline JcSSR-32 & $\begin{array}{l}\text { F: TCTTTTGACTACCCAATGACCA } \\
\text { R: TCGTGCTAGAGTTGGTTGAAAT }\end{array}$ & 60 & 186-192 & 4 & 0.644 & 0.563 & 0.5595 & 0.126 & 0.565 \\
\hline JcSSR-33 & $\begin{array}{l}\text { F: GAGAACGTGGAGAGTTAAAA } \\
\text { R: TGGATGGATCATTGTCTG }\end{array}$ & 55 & $237-297$ & 16 & 0.906 & 0.778 & 0.9576 & 0.142 & 0.886 \\
\hline JcSSR-34 & $\begin{array}{l}\text { F: CTAAAAGTTTTGGACATTTTTAC } \\
\text { R: TTAATGGAAGCACTCCTGTAAT }\end{array}$ & 55 & 174-186 & 4 & 0.118 & 0.082 & 0.1529 & 0.311 & 0.114 \\
\hline JcSSR-35 & $\begin{array}{l}\text { F: GAGTTTGGTTGGATGGATA } \\
\text { R: TAGAACCGCAGCAGAAA }\end{array}$ & 54 & $172-208$ & 10 & 0.889 & 0.769 & 0.9896 & 0.135 & 0.856 \\
\hline JcSSR-36 & $\begin{array}{l}\text { F: GCACTCAAAAGTGTTTCCCAA } \\
\text { R: ATCACACCAAAGAAGAATGCAG }\end{array}$ & 59 & $163-187$ & 4 & 0.699 & 0.636 & 0.9487 & 0.090 & 0.620 \\
\hline JcSSR-37 & $\begin{array}{l}\text { F: TGCAGGCACACCAAACAA } \\
\text { R: TTTCGATGGAATCTAGGTTAGTCA }\end{array}$ & 58 & $269-299$ & 11 & 0.873 & 0.761 & $0.0000^{*}$ & 0.128 & 0.847 \\
\hline JcSSR-38a & $\begin{array}{l}\text { F: TGAAGCTGTCTTTGATTGAGGAA } \\
\text { R: TCTTGGGATCGTCTACAAGGTCT }\end{array}$ & 61 & $209-213$ & 3 & 0.521 & 0.469 & 0.2779 & 0.098 & 0.431 \\
\hline JcSSR-38b & $\begin{array}{l}\text { F: ATCAGCAACTCCTTAATGCCAA } \\
\text { R: CAACTCAAAAGTTCAGCCCAAA }\end{array}$ & 59 & $153-157$ & 3 & 0.537 & 0.461 & 0.2991 & 0.081 & 0.441 \\
\hline JcSSR-39 & $\begin{array}{l}\text { F: TTTTAACATATTGGGTTGAATTTT } \\
\text { R: TTTTGCATAAATAACAGAAGGT }\end{array}$ & 58 & 115 & 1 & - & - & - & - & - \\
\hline JcSSR-40 & $\begin{array}{l}\text { F: AGTGGTGGTTGTGCTCATAGTG } \\
\text { R: GTGGGTCACGCTATCCATATCT }\end{array}$ & 61 & 194-218 & 11 & 0.877 & 0.723 & 0.1786 & 0.175 & 0.853 \\
\hline JcSSR-41 & $\begin{array}{l}\text { F: GTACGAGCGTGGACTAACT } \\
\text { R: GACATTACAAATATTTTGAAACG }\end{array}$ & 52 & $157-179$ & 9 & 0.800 & 0.700 & 0.9525 & 0.125 & 0.758 \\
\hline JcSSR-42 & $\begin{array}{l}\text { F: TGCATCAACACATCCATTCTTT } \\
\text { R: GAGTGGGAAGTAGCATTTGGAA }\end{array}$ & 57 & $138-142$ & 3 & 0.375 & 0.298 & 0.0379 & 0.206 & 0.338 \\
\hline
\end{tabular}

*Significant at the 5\% significance level $(\mathrm{P}>0.0029)$. Forty-nine samples were genotyped; $N_{\mathrm{A}}=$ number of alleles at each locus; $H_{\mathrm{E}}=$ expected heterozygosity; $H_{\mathrm{O}}=$ observed heterozygosity; HWE = deviation from Hardy-Weinberg equilibrium; $F_{\text {IS }}=$ inbreeding coefficient PIC = polymorphic information content. 

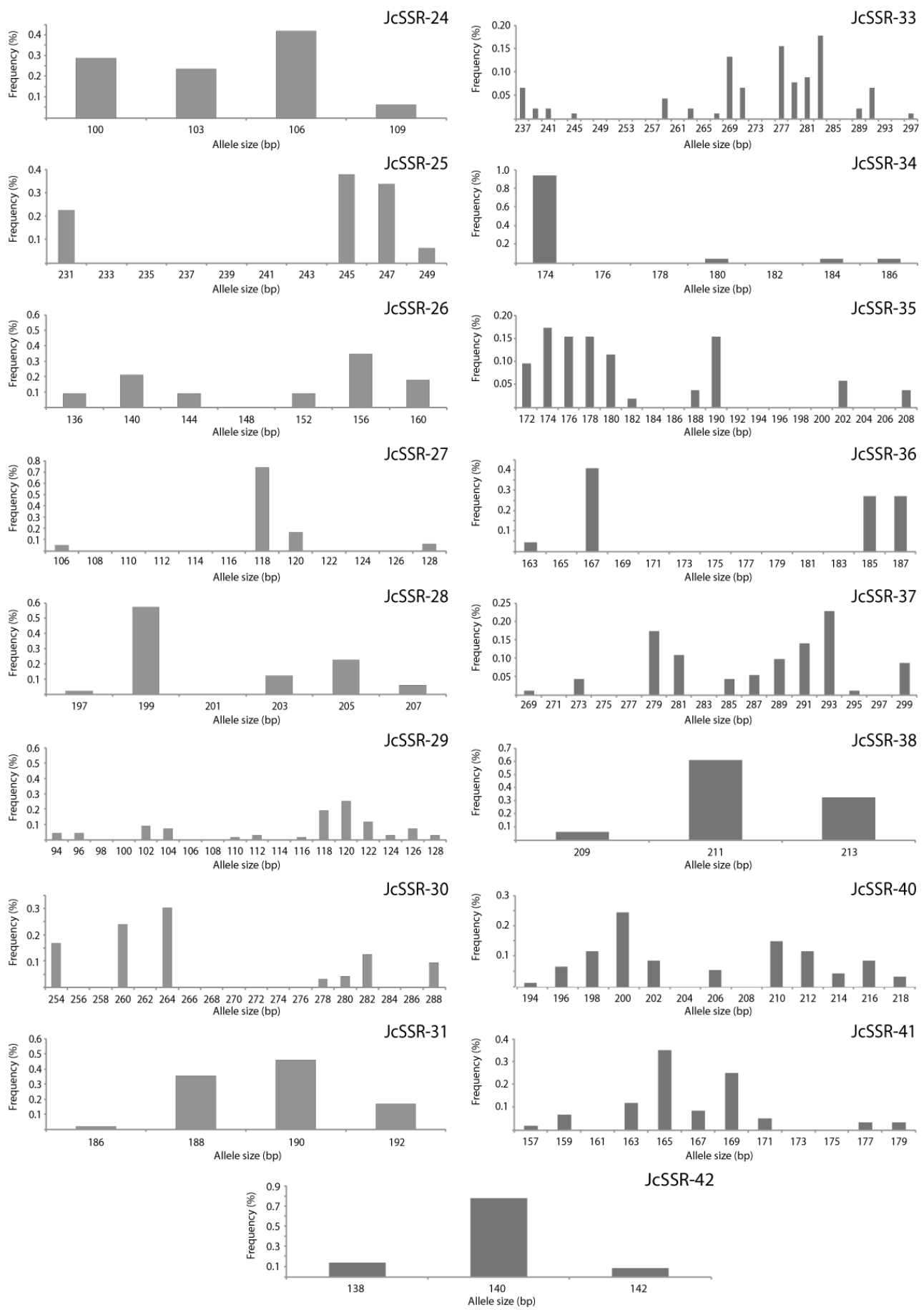

Figure 1. Distributions of allele frequencies at the 17 polymorphic microsatellite loci in Jatropha curcas populations. 


\section{ACKNOWLEDGMENTS}

Research supported by the Brazilian Government, the Brazilian Ministry of Science and Technology (MCT/FINEP, BRJatropha Project), the Bank of Northeastern Brazil (BNB/ ETENE/FUNDECI), and the Brazilian Agricultural Research Corporation (EMBRAPA).

\section{REFERENCES}

Amos W (1999). A Comparative Approach to the Study of Microsatellite Evolution. In: Microsatellites: Evolution and Applications (Goldstein DB and Schlotterer C, eds.). Oxford University Press, Oxford, 67-79.

Arruda FP, Beltrão NEM, Andrade AP, Pereira WE, et al. (2004). Cultivo de pinhão manso (Jatropha curcas L.) como alternativa para o semi-árido nordestino. Rev. Bras. Oleag. Fibr. 8: 789-799.

Berchmans HJ and Hirata S (2008). Biodiesel production from crude Jatropha curcas L. seed oil with a high content of free fatty acids. Bioresour. Technol. 99: 1716-1721.

Botstein D, White RL, Skolnick M and Davis RW (1980). Construction of a genetic linkage map in man using restriction fragment length polymorphisms. Am. J. Hum. Genet. 32: 314-331.

Castelo AT, Martins W and Gao GR (2002). TROLL - tandem repeat occurrence locator. Bioinformatics 18: 634-636.

DeWoody JA, Honeycutt RL and Skow LC (1995). Microsatellite markers in white-tailed deer. J. Hered. 86: 317-319.

Dias LA, Missio RF and Dias DC (2012). Antiquity, botany, origin and domestication of Jatropha curcas (Euphorbiaceae), a plant species with potential for biodiesel production. Genet. Mol. Res. 11: 2719-2728.

Ellegren H (2004). Microsatellites: simple sequences with complex evolution. Nat. Rev. Genet. 5: 435-445.

Emeasor KC, Ogbuji RO and Emosairue SO (2005). Insecticidal activity of some seed powders against Callosobruchus maculatus (F.) (Coleoptera: Bruchidae) on stored cowpea. J. Plant Dis. Protect. 112: 80-87.

Fairless D (2007). Biofuel: the little shrub that could - maybe. Nature 449: 652-655.

Goudet J (1995). FSTAT (version 1.2): a computer program to calculate F-statistics. J. Hered. 86: 485-486.

Guo BY, Qi PZ, Zhu AY, Lv ZM, et al. (2013). Isolation and characterization of new polymorphic microsatellite markers from the cuttlefish Sepiella maindroni (Cephalopoda; Sepiidae). Genet. Mol. Res. 12: 2376-2379.

Kalinowski ST, Taper ML and Marshall TC (2007). Revising how the computer program CERVUS accommodates genotyping error increases success in paternity assignment. Mol. Ecol. 16: 1099-1106.

Nambisan P (2007). Biotechnological intervention in Jatropha for biodiesel production. Curr. Sci. 93: 1347-1348.

Qi PZ, Xie CX, Guo BY, Wu CW, et al. (2013). Development of new polymorphic microsatellite markers in topmouth culter (Culter alburnus) and determination of their applicability in Culter mongolicus. Genet. Mol. Res. 12: 1761-1765.

Raymond M and Rousset F (1995). Genepop (version 1.2): population genetics software for exact tests and ecumenicism. J. Hered. 86: 248-249.

Rozen S and Skaletsky HJ (2000). Primer3 on the WWW for General Users and for Biologist Programmers. In: Bioinformatics Methods and Protocols (Methods in Molecular Biology) (Krawetz S and Misener S, eds.). Humana Press, Totowa, 365-386.

Sato S, Hirakawa H, Isobe S, Fukai E, et al. (2011). Sequence analysis of the genome of an oil-bearing tree, Jatropha curcas L. DNA Res. 18: 65-76.

Thomas R, Sah NK and Sharma PB (2008). Therapeutic biology of Jatropha curcas: a mini review. Curr. Pharm. Biotechnol. 9: 315-324.

Van Oosterhout C, Hutchinson WF, Shipley P and Wills DPM (2004). Micro-Checker: Software for identifying and correcting genotyping errors in microsatellite data. Mol. Ecol. Notes 4: 535-538. 